\title{
Correction to: Protocol for the Emory University African American Vaginal, oral, and gut microbiome in pregnancy cohort study
}

Elizabeth J. Corwin ${ }^{1 *}$, Carol J. Hogue ${ }^{2}$, Bradley Pearce ${ }^{2}$, Cherie C. Hill ${ }^{3}$, Timothy D. Read ${ }^{4}$, Jennifer Mulle ${ }^{4}$ and Anne L. Dunlop ${ }^{5}$

\section{Correction}

Following publication of the original article [1], the authors pointed out that the Methods included one step that is no longer necessary but which was inadvertently carried over from an earlier protocol.

\footnotetext{
Author details

'Emory University School of Nursing, 1520 Clifton Rd, Atlanta, GA 30322, USA. ${ }^{2}$ Emory University Rollins School of Public Health, Atlanta, GA, USA.

${ }^{3}$ Department of Gynecology and Obstetrics, Emory University, Atlanta, GA, USA. ${ }^{4}$ Emory University School of Medicine, Atlanta, GA, USA. ${ }^{5}$ Emory University School of Nursing and School of Medicine, Atlanta, GA, USA.
}

Published online: 27 November 2017

\section{Reference}

1. Elizabeth J. Corwin, Carol J. Hogue, Bradley Pearce, Cherie C. Hill, Timothy D. Read, Jennifer Mulle and Anne L. Dunlop. BMC Pregnancy and Childbirth (2017) 17:161. DOI 10.1186/s12884-017-1357-x

*Correspondence: ejcorwi@emory.edu

${ }^{1}$ Emory University School of Nursing, 1520 Clifton Rd, Atlanta, GA 30322, USA 